\title{
BULK UNIVERSALITY FOR WIGNER HERMITIAN MATRICES WITH SUBEXPONENTIAL DECAY
}

\author{
LÁszló Erdős, José Ramírez, Benjamin Schlein, Terence Tao, Van Vu, \\ AND HORNG-TZER YAU
}

\begin{abstract}
In this paper, we consider the ensemble of $n \times n$ Wigner Hermitian matrices $H=\left(h_{\ell k}\right)_{1 \leq \ell, k \leq n}$ that generalize the Gaussian unitary ensemble (GUE). The matrix elements $h_{k \ell}=\bar{h}_{\ell k}$ are given by $h_{\ell k}=n^{-1 / 2}\left(x_{\ell k}+\sqrt{-1} y_{\ell k}\right)$, where $x_{\ell k}$, $y_{\ell k}$ for $1 \leq$ $\ell<k \leq n$ are i.i.d. random variables with mean zero and variance $1 / 2, y_{\ell \ell}=0$ and $x_{\ell \ell}$ have mean zero and variance 1 . We assume the distribution of $x_{\ell k}, y_{\ell k}$ to have subexponential decay. In [3], four of the authors recently established that the gap distribution and averaged $k$-point correlation of these matrices were universal (and in particular, agreed with those for GUE) assuming additional regularity hypotheses on the $x_{\ell k}, y_{\ell k}$. In [7], the other two authors, using a different method, established the same conclusion assuming instead some moment and support conditions on the $x_{\ell k}, y_{\ell k}$. In this short note we observe that the arguments of [3] and [7] can be combined to establish universality of the gap distribution and averaged $k$-point correlations for all Wigner matrices (with subexponentially decaying entries), with no extra assumptions.
\end{abstract}

\section{Introduction}

This paper is concerned with the bulk eigenvalue statistics of large Hermitian Wigner random matrices, which we now pause to define.

Definition 1 (Wigner matrices). Let $n$ be a large number. A Wigner Hermitian matrix (of size $n$ ) is defined as a random Hermitian $n \times n$ matrix $H=H_{n}$ with upper triangular complex entries $h_{\ell k}=n^{-1 / 2}\left(x_{\ell k}+\sqrt{-1} y_{\ell k}\right)$ for $1 \leq \ell<k \leq n$ and diagonal real entries $h_{\ell \ell}=n^{-1 / 2} x_{\ell \ell}$ for $1 \leq \ell \leq n$, where

- For $1 \leq \ell<k \leq n$, the $x_{\ell k}, y_{\ell k}$ are i.i.d. copies of a real random variable $x$ with mean zero and variance $1 / 2$.

- For $1 \leq \ell \leq n$, the $x_{\ell \ell}$ are i.i.d. copies of a real random variable $\tilde{x}$ with mean zero and variance 1 .

- $x, \tilde{x}$ have subexponential decay, i.e., there are constants $C, C^{\prime}$ such that $\mathbf{P}\left(|x| \geq t^{C}\right) \leq \exp (-t), \mathbf{P}\left(|\tilde{x}| \geq t^{C}\right) \leq \exp (-t)$, for all $t \geq C^{\prime}$.

We refer to $x$ as the atom distribution of $H$. An important special case of a Wigner Hermitian matrix is the Gaussian unitary ensemble (GUE), in which $x, \tilde{x}$ are Gaussian random variables with mean zero and variance $1 / 2,1$ respectively.

Let $\lambda_{1} \leq \ldots \leq \lambda_{n}$ be the eigenvalues of a Wigner Hermitian matrix $H_{n}$, and let $p_{n}\left(x_{1}, \ldots, x_{n}\right)$ be the associated (symmetric) density function, normalized to have

Received by the editors June 24, 2009. 
total mass 1 . For any $1 \leq k \leq n$, the $k$-point correlation function $p_{n}^{(k)}\left(x_{1}, \ldots, x_{k}\right)$ is defined by the formula

$$
p_{n}^{(k)}\left(x_{1}, \ldots, x_{k}\right):=\int_{\mathbb{R}^{n-k}} p_{n}\left(x_{1}, \ldots, x_{n}\right) d x_{k+1} \ldots d x_{n} .
$$

Here we follow the convention that the correlation functions are $L^{1}$-normalized. Other conventions are also used in the literature. e.g. the correlation functions $R_{k}$ used in $[1,4]$ are related to $p_{n}^{(k)}$ via $R_{k}=\frac{n !}{(n-k) !} p_{n}^{(k)}$.

The main results of this note are as follows.

Theorem 2 (Universality of averaged correlation function). Fix $\varepsilon>0$ and $u$ such that $-2<u-\varepsilon<u+\varepsilon<2$. Let $k \geq 1$ and let $f: \mathbb{R}^{k} \rightarrow \mathbb{R}$ be a continuous, compactly supported function, and let $H=H_{n}$ be a Wigner random matrix. Then the quantity

$$
\begin{aligned}
\frac{1}{2 \varepsilon} \int_{u-\varepsilon}^{u+\varepsilon} & \int_{\mathbb{R}^{k}} f\left(\alpha_{1}, \ldots, \alpha_{k}\right) \\
& \times \frac{1}{\left[\rho_{s c}\left(u^{\prime}\right)\right]^{k}} p_{n}^{(k)}\left(u^{\prime}+\frac{\alpha_{1}}{n \rho_{s c}\left(u^{\prime}\right)}, \ldots, u^{\prime}+\frac{\alpha_{k}}{n \rho_{s c}\left(u^{\prime}\right)}\right) d \alpha_{1} \ldots d \alpha_{k} d u^{\prime}
\end{aligned}
$$

converges as $n \rightarrow \infty$ to

$$
\int_{\mathbb{R}^{k}} f\left(\alpha_{1}, \ldots, \alpha_{k}\right) \operatorname{det}\left(K\left(\alpha_{i}, \alpha_{j}\right)\right)_{i, j=1}^{k} d \alpha_{1} \ldots d \alpha_{k}
$$

where $K(x, y)$ is the Dyson sine kernel

$$
K(x, y):=\frac{\sin (\pi(x-y))}{\pi(x-y)}
$$

and $\rho_{s c}(u)$ is the Wigner semicircle distribution

$$
\rho_{s c}(u):=\frac{1}{2 \pi}\left(4-u^{2}\right)_{+}^{1 / 2} .
$$

Theorem 3 (Universality of averaged eigenvalue gap). Fix $u$ such that $-2<u<2$, choose $k \geq 1$ and $s>0$, and let $H$ be a Wigner random matrix. Then the expectation value of the empirical gap distribution

$$
\Lambda_{n}(u, s, \varepsilon)=\frac{1}{2 \varepsilon n \rho_{s c}(u)} \#\left\{1 \leq j<n: \lambda_{j+1}-\lambda_{j} \leq \frac{s}{n \rho_{s c}(u)} ;\left|\lambda_{j}-u\right| \leq \varepsilon\right\}
$$

satisfies

$$
\lim _{\varepsilon \rightarrow 0} \lim _{n \rightarrow \infty} \mathbf{E} \Lambda_{n}(u, s, \varepsilon)=\int_{0}^{s} \frac{d^{2}}{d \alpha^{2}} \operatorname{det}\left(1-\mathcal{K}_{\alpha}\right) d \alpha,
$$

where $\mathcal{K}_{\alpha}$ is the integral operator on $L^{2}((0, \alpha))$ whose kernel is the Dyson sine kernel (2).

We now briefly recall the previous partial results towards these theorems. In the case of GUE, the proofs of Theorems 2, 3 can be found in [5]. These proofs relied heavily on the explicit formula for the $k$-point correlation functions in the GUE case. An important extension was made by Johansson [4], who established the above theorems in Gaussian divisible case when the matrix $H$ took the form $\hat{H}+a V$, where $\hat{H}$ is another Wigner matrix, $V$ is a GUE matrix independent of $\hat{H}$ and $a>0$ is an arbitrary fixed constant independent of $n$. This proof also requires the use of an 
explicit formula, however, its analysis is much more delicate than in the GUE case. By a simple rescaling, it is clear that Johansson's result extends to Wigner matrices of the form

$$
H=e^{-t / 2} \hat{H}+\left(1-e^{-t}\right)^{1 / 2} V,
$$

where $\hat{H}$ and $V$ are as above, and the time $t>0$ is fixed independent of $n$. One can view $H$ as the evolution of $\hat{H}$ under an Ornstein-Uhlenbeck process of time $t$.

In [3], four of the authors extended Johansson's result to obtain Theorems 2 and 3 for matrices of the form (5) with a short time $t=n^{-1+\delta}$ for any fixed $\delta>0$. An important ingredient was the convergence of the density of the eigenvalues to the semicircle law (3) on $O\left(n^{-1+\delta}\right)$ scales which was previously established in [2]. Combining the result for Wigner matrices of the form (5) for time $t=n^{-1+\delta}$ with a time-reversal argument, Theorems 2 and 3 were also obtained for Wigner matrices $H$ with no Gaussian component, under the assumption that the atom distribution of $H$ has a probability density $h(x)=e^{-g(x)}$, where $g \in C^{6}(\mathbb{R})$ grows at most polynomially fast at infinity and $h(x) \leq C e^{-c|x|}$. For more details, we refer to [3, Section 1].

In [7], the other two authors introduced a different way to study the local eigenvalue statistics of random matrices, based on a variant of Lindeberg replacement strategy. This enables one to compare the statistics of a general Wigner matrix to those of a Johansson matrix, or any random matrix where the desired statistics is known, given that some moment condition is satisfied. As an application, Theorems 2, 3 were established in [7] under the additional hypothesis that the atom distribution $x$ had vanishing third moment and was supported on at least three points. For more details, we refer to [7, Section 1].

In this note, we observe that one can combine the arguments from [3] with the arguments of [7] to eliminate the regularity hypotheses from the former and the moment and support hypotheses from the latter. The basic idea is simple and can be described as follows. The main result of [7] (see [7, Theorem 15]) allows one to compare the local statistics of two Wigner hermitian matrices whose atom variables $\xi, \xi^{\prime}$ have the same third and fourth moments, namely $\mathbf{E} \xi^{3}=\mathbf{E} \xi^{\prime 3}$ and $\mathbf{E} \xi^{4}=\mathbf{E} \xi^{\prime 4}$. A closer look reveals that it is enough to require that the differences $\left|\mathbf{E} \xi^{3}-\mathbf{E} \xi^{\prime 3}\right|$ and $\left|\mathbf{E} \xi^{4}-\mathbf{E} \xi^{\prime 4}\right|$ are sufficiently small (in term of $n$ ). This allows us to compare the original matrix $H$ with a Johansson-type matrix (5) with $t=n^{-1+\delta}$ for some small $\delta$, instead of comparing with a Johansson matrix with $t=\Theta(1)$ as done in [7]. On the other hand, arguments from [3] guarantee that such a Johansson matrix has the desired statistics.

One defect of our method is that the statistics need to be averaged over a spatial scale $\varepsilon$ which is independent of $n$ before universality is established. This appears to be a largely technical condition, arising from the lack of good concentration results for individual eigenvalues $\lambda_{i}$ for matrices of the form (5) (in particular, we do not know how to eliminate the vanishing third moment hypothesis from [7, Theorem 29]). However, if we reimpose the hypothesis that the third moment of the atom distribution vanishes (i.e., $\mathbf{E} x^{3}=0$ ), we can remove the averaging in Theorem 2, thus refining [7, Theorem 11] by removing the condition that the support must have at least 3 points (see Section 3). 


\section{Proof of theorems}

We now prove Theorem 2 . We fix $u, \varepsilon, f, H$; by a limiting argument we may take $f$ to be smooth. We will always assume $n$ to be sufficiently large depending on these parameters. Using the exponential decay of $x$ and a standard truncation argument (see [7, Section 3.1]) we may assume that

$$
x=O\left(\log ^{O(1)} n\right)
$$

almost surely. (Note that the Gaussian random variable $N(0,1 / 2)$ does not quite obey this bound, but can be modified on a set of extremely small probability to do so whenever necessary, so we may effectively assume that (6) holds in this case also.)

We introduce the modified Wigner matrix

$$
H^{\prime}:=e^{-t / 2} H+\left(1-e^{-t}\right)^{1 / 2} V,
$$

where $t:=n^{-1+\delta}$ and $V$ is a GUE matrix independent of $H$. We will choose $\delta=0.01$ (say), but the proof below actually requires only $\delta<1 / 2$.

Observe that $H^{\prime}$ is another Wigner random matrix, with atom distribution $x^{\prime}$ given by

$$
x^{\prime}:=e^{-t / 2} x+\left(1-e^{-t}\right)^{1 / 2} g
$$

where $g \equiv N(0,1 / 2)$ is a Gaussian random variable independent of $x$. In particular we have the moment comparision bounds

$$
\mathbf{E}\left(x^{\prime}\right)^{j}=\mathbf{E} x^{j}
$$

for $j=0,1,2$, and

$$
\mathbf{E}\left(x^{\prime}\right)^{j}=\mathbf{E} x^{j}+O\left(n^{-1+0.01}\right)
$$

for $j=3,4$

Let $\left(p_{n}^{\prime}\right)^{(k)}$ be the $k$-point correlation function associated to $H^{\prime}$. The first step is to show that Theorem 2 is valid for $H^{\prime}$ :

Proposition 4. The quantity

$$
\begin{aligned}
\frac{1}{2 \varepsilon} \int_{u-\varepsilon}^{u+\varepsilon} & \int_{\mathbb{R}^{k}} f\left(\alpha_{1}, \ldots, \alpha_{k}\right) \\
& \times \frac{1}{\left[\rho_{s c}\left(u^{\prime}\right)\right]^{k}}\left(p_{n}^{\prime}\right)^{(k)}\left(u^{\prime}+\frac{\alpha_{1}}{n \rho_{s c}\left(u^{\prime}\right)}, \ldots, u^{\prime}+\frac{\alpha_{k}}{n \rho_{s c}\left(u^{\prime}\right)}\right) d \alpha_{1} \ldots d \alpha_{k} d u^{\prime}
\end{aligned}
$$

converges as $n \rightarrow \infty$ to

$$
\int_{\mathbb{R}^{k}} f\left(\alpha_{1}, \ldots, \alpha_{k}\right) \operatorname{det}\left(K\left(\alpha_{i}, \alpha_{j}\right)\right)_{i, j=1}^{k} d \alpha_{1} \ldots d \alpha_{k} .
$$

Proof. By the dominated convergence theorem, it suffices to show that

(9)

$$
\begin{aligned}
\lim _{n \rightarrow \infty} \int_{\mathbb{R}^{k}} f\left(\alpha_{1}, \ldots, \alpha_{k}\right) & \frac{1}{\left[\rho_{s c}(u)\right]^{k}}\left(p_{n}^{\prime}\right)^{(k)}\left(u+\frac{\alpha_{1}}{n \rho_{s c}(u)}, \ldots, u+\frac{\alpha_{k}}{n \rho_{s c}(u)}\right) d \alpha_{1} \ldots d \alpha_{k} \\
& =\int_{\mathbb{R}^{k}} f\left(\alpha_{1}, \ldots, \alpha_{k}\right) \operatorname{det}\left(K\left(\alpha_{i}, \alpha_{j}\right)\right)_{i, j=1}^{k} d \alpha_{1} \ldots d \alpha_{k} .
\end{aligned}
$$

for every fixed $-2<u<2$. The dominated convergence theorem can be applied since the integral on the l.h.s. of (9) can be bounded by $C_{k} \mathbf{E} N_{I}^{k}$, where $N_{I}$ is 
the number of eigenvalues in an interval $I$ of size $C / n$ about $u$. The boundedness of $\mathbf{E} N_{I}^{k}$ follows from [2, Theorem 5.1]. Originally this result was stated for atom distribution with Gaussian decay, but the proof can be modified for random variables with subexponential decay and satisfying (6).

Eq. (9) is proven in Proposition 3.1 of [3]. The proof is based on an explicit integral representation for the marginal $\left(p_{n}^{\prime}\right)^{(k)}$. The only assumption used to prove (9) in [3] is the asymptotic validity of the local semicircle law for the density of the eigenvalues of the Wigner matrix $H$ (with no Gaussian part) on scales of order $n^{-1+\delta}$. More precisely, if

$$
m_{n}(z)=\frac{1}{n} \operatorname{trace} \frac{1}{H-z}, \quad \text { and } \quad m_{s c}(z)=\int \frac{d s \varrho_{s c}(s)}{s-z}
$$

denote the Stieltjes transforms of the empirical eigenvalue distribution and of the semicircle law, respectively, then we need to know $[3$, Eq. (3.9)] that for any $\kappa, \delta>0$ there exist constants $c, C>0$ such that

$$
\mathbf{P}\left(\sup _{E \in(-2+\kappa ; 2-\kappa)}\left|m_{n}(E+i \eta)-m_{s c}(E+i \eta)\right| \geq \varepsilon\right) \leq C e^{-c \varepsilon \sqrt{n \eta}}
$$

for $n^{-1+\delta} \leq \eta \leq 1$ and for all $\varepsilon>0$ sufficiently small.

Eq. (10) has been established in [2] under the assumption of Gaussian decay of the atom distribution, that is assuming that $\mathbf{E} e^{\beta x^{2}}<\infty$, for some $\beta>0$. To reduce this assumption to the desired sub-exponential decay assumption, we can use a result in [7] (see the paragraph following [7, Lemma 60]) which proved (10) under this weaker assumption with a weaker probability bound $\exp (-\omega(\log n))$, where $\omega(\log n)$ denotes a quantity whose ratio with $\log n$ goes to infinity as $n \rightarrow \infty$. One can also follow the ideas in Section 5 of [3], where it is shown that the Gaussian decay can be relaxed to exponential decay (at the expense of a slightly weaker probability bound). These ideas can be further refined to deal with sub-exponential decay.

Following the proof of Proposition 3.1 in [3], it is clear that the argument holds using this weaker bound on the probability as well. This concludes the proof of Proposition 4.

To conclude the proof of Theorem 2, it thus suffices to show that the expression (1) only changes by $o(1)$ when we replace $H$ with $H^{\prime}$, where we use $o(1)$ to denote an expression that goes to zero as $n \rightarrow \infty$.

From the definition of $p_{n}^{(k)}$, we can rewrite (1) as

$$
\frac{1}{n} \sum_{1 \leq i_{1}, \ldots, i_{k} \leq n} \mathbf{E} G\left(n \lambda_{i_{1}}, \ldots, n \lambda_{i_{k}}\right)
$$

where

$$
G\left(y_{1}, \ldots, y_{k}\right):=\frac{n}{2 \varepsilon} \int_{u-\varepsilon}^{u+\varepsilon} \frac{d u^{\prime}}{\left[\rho_{s c}\left(u^{\prime}\right)\right]^{k}} f\left(\rho_{s c}\left(u^{\prime}\right)\left(y_{1}-n u^{\prime}\right), \ldots, \rho_{s c}\left(u^{\prime}\right)\left(y_{k}-n u^{\prime}\right)\right) .
$$

On the other hand, by [7, Proposition 61] (or [2, Theorem 5.1]), taking advantage of the hypothesis (6), we see that, with probability $1-O\left(n^{-100}\right)$ (say),

$$
\#\left\{1 \leq i \leq n: \lambda_{i} \in I\right\} \leq \widetilde{C} n|I|
$$


for all intervals $I \subset[u-\varepsilon, u+\varepsilon]$ with $|I| \geq \log ^{C} n / n$, for some sufficiently large absolute constant $C, \widetilde{C}$, where $\widetilde{C}$ can depend on $u$ and $\varepsilon$. Because of this, and the compact support of $f$, we can restrict the summation in (11) to those $i_{1}, \ldots, i_{k}$ for which $i_{j}=i_{1}+O\left(\log ^{O(1)} n\right)$ for all $1 \leq j \leq k$, while only paying an acceptable error of $o(1)$. In particular, there are now only $O\left(n \log ^{O(1)} n\right)$ summands in (11). Similarly, using the convergence to the Wigner semicircular law (see e.g. [6]) we may restrict $i_{1}, \ldots, i_{k}$ to the interval $[\delta n,(1-\delta) n]$ for some fixed $\delta>0$ independent of $n$.

A routine computation shows that $G$ enjoys the derivative bounds

$$
\left|\nabla^{j} G\left(y_{1}, \ldots, y_{k}\right)\right|=O(1)
$$

for all $y_{1}, \ldots, y_{k}$ and $0 \leq j \leq 5$, where the implied constant in the $O()$ notation can depend on $u, \varepsilon, f$.

Suppose first that the random variables $x, x^{\prime}$ matched to fourth order. One could then apply the four moment theorem in [7, Theorem 15] to conclude that, for each $i_{1}, \ldots, i_{k} \in[\delta n,(1-\delta) n]$, the quantity $\mathbf{E} G\left(n \lambda_{i_{1}}, \ldots, n \lambda_{i_{k}}\right)$ changes by $O\left(n^{-c}\right)$ for some absolute constant $c>0$, uniformly in $i_{1}, \ldots, i_{k}$, when $H$ is replaced by $H^{\prime}$. Inserting this into (11), we obtain the desired claim.

In our situation, $x$ and $x^{\prime}$ do not match exactly to fourth order, but only to second order. However, we have approximate matching to third and fourth order thanks to (8), and this is enough to recover the conclusions of the four moment theorem. Indeed, an inspection of the proof of [7, Theorem 15] in [7, Section 3.3] reveals that the matching moments condition is used in only one place, namely to show that the expectation of an expression of the form

$$
F(0)+\nabla F(0)\left(h_{\ell k}\right)+\ldots+\frac{1}{4 !} \nabla^{4} F(0)\left(h_{\ell k}, \ldots, h_{\ell k}\right)+O\left(n^{-5 / 2+o(1)}\right)
$$

only changes by at most $n^{-2-0.1}$ (say) when one replaces $h_{\ell k}=n^{-1 / 2}\left(x_{\ell k}+\sqrt{-1} y_{\ell k}\right)$ with $h_{\ell k}^{\prime}:=n^{-1 / 2}\left(x_{\ell k}+\sqrt{-1} y_{\ell k}\right)$, where $1 \leq \ell<k \leq n$, and $F: \mathbb{C} \rightarrow \mathbb{R}$ is a function obeying the derivative bounds

$$
\left|\nabla^{j} F(0)\right| \leq C n^{-j+o(1)}
$$

for $0 \leq j \leq 4$. But the expectation of the first three terms of this expansion does not change when replacing $h_{\ell k}$ with $h_{\ell k}^{\prime}$ thanks to (7), and the next two terms change by $O\left(n^{-3+o(1)} n^{3 / 2} n^{-1+0.01}\right)$ and $O\left(n^{-4+o(1)} n^{4 / 2} n^{-1+0.01}\right)$ respectively, and the claim follows.

Remark 5. The reader will observe that there is some room to spare here regarding powers of $n$. Indeed, a more careful examination of the argument reveals that one can sharpen Theorem 2 by not keeping $\varepsilon$ fixed with respect to $n$, but instead letting it decay as $\varepsilon=n^{-1 / 2+\delta}$ for any $0<\delta<1 / 2$. This should not be the optimal result, however; the correct scale should be $O\left(n^{-1+\delta}\right.$ ) (and in fact one should not need to average in $u$ at all). Unfortunately, our methods so far can only achieve this by putting more moment or regularity hypotheses on the distribution $x$, see for instance Section 3.

We now sketch the proof of Theorem 3. The first step is to prove that the empirical gap distribution $\Lambda_{n}^{\prime}$ of the matrix $H^{\prime}$ satisfies the equation (4). This follows from the same argument used in the proof of of Theorem 1.2 in [3]. The only difference is that 
the parameter $\varepsilon$ in (4) was replaced by $n^{-1+\delta}$ (with some $0<\delta<1$ ) in [3]. Since the change of the density $\varrho_{s c}\left(u^{\prime}\right)$ vanishes as $\varepsilon \rightarrow 0$, the same proof applies to the current setting, using that the limiting function on the r.h.s. of (4) is continuous in $s$.

In the second step we deduce (4) for $H$ by using that the expected values of observables of the form (11) are asymptotically the same for $H$ and $H^{\prime}$. By the exclusion-inclusion principle, $\Lambda_{n}$ can be written as an alternating series of expressions in the form (11) with functions $G$ which are products of characteristic functions of intervals of length one or larger. These characteristic functions can be approximated by bounded smooth functions in $L^{1}$-sense with arbitrary precision $\kappa$. Controlling the alternating series by its last term, letting first $n \rightarrow \infty$ then $\kappa \rightarrow 0$ and using the continuity in $s$ of the limiting function, we obtain (4) for $H$ as well.

\section{Concluding remarks}

If one assumes the additional third moment condition $\mathbf{E} x^{3}=0$ on the moment condition, then it was shown in [7, Theorem 29] that the eigenvalues $\lambda_{i}$ with $i \in$ $[\delta n,(1-\delta) n]$ obeyed the localization property

$$
\lambda_{i}=t\left(\frac{i}{n}\right)+O\left(n^{-1+c}\right)
$$

with probability $1-O\left(n^{-C}\right)$ for any $C, c>0$ (and $n$ sufficiently large depending on $C, c, \delta)$, where $t(a)$ was defined by the formula

$$
a:=\int_{-2}^{t(a)} \rho_{s c}(x) d x
$$

Because of this localization, it is possible to eliminate the averaging over $u^{\prime}$ in Theorem 2, and to reduce the averaging in Theorem 3 to an interval of $O\left(n^{\delta}\right)$ eigenvalues rather than $O(n)$, by combining the above arguments with those used to prove $[7$, Theorems 9,11]. The net effect of this is to eliminate the hypothesis in those theorems that $x$ is supported on at least three points. Thus, for instance, we can take $x$ to be the Bernoulli variable that takes values \pm 1 with an equal probability $1 / 2$.

If the third moment condition from [7, Theorem 29] could be omitted, then one could similarly reduce the averaging required for Theorems 2,3 even when the third moment was non-zero. To do so, however, would require either a relaxation of the moment conditions needed in [7, Theorem 15], or else an analogue of [7, Theorem 29] for the matrices $H^{\prime}$ studied here. It seems plausible that at least one of these two approaches could eventually be made to work, but this does not appear to be easily deducible from the existing literature.

Another remark is that our theorems hold for a more general model of random matrices, where the real and imaginary parts of the entries are not necessarily independent. The reason is that [7, Theorem 15] (and its variant used here) do not require this assumption (see also the end of $[7$, Section 1]).

\section{Acknowledgements}

L. Erdős is partially supported by SFB-TR 12 Grant of the German Research Council. T. Tao is is supported by a grant from the MacArthur Foundation, by NSF grant DMS-0649473, and by the NSF Waterman award. V. Vu is supported by 
NSF grant DMS-0901216 and DOD grant AFOSAR-FA-9550-09-1-0167. H.-T. Yau is partially supported by NSF grants DMS-0757425, DMS-0804279.

\section{References}

[1] P. Deift, Orthogonal polynomials and random matrices: a Riemann-Hilbert approach. Courant Lecture Notes in Mathematics, 3. New York University, Courant Institute of Mathematical Sciences, New York; American Mathematical Society, Providence, RI, 1999.

[2] L. Erdős, B. Schlein and H-T. Yau, Wegner estimate and level repulsion for Wigner random matrices. Int. Math. Res. Notices. 2010, No. 3, 436-479 (2010)

[3] L. Erdős, S. Péché, J. Ramírez, B. Schlein and H-T. Yau, Bulk universality for Wigner matrices. Commun. Pure Appl. Math. Volume 63, Issue 7, p 895-925 (2010).

[4] K. Johansson, Universality of the local spacing distribution in certain ensembles of Hermitian Wigner matrices, Comm. Math. Phys. 215 (2001), no. 3, 683-705.

[5] M.L. Mehta, Random matrices and the statistical theory of energy levels, Academic Press, New York, NY, 1967.

[6] L. Pastur, On the spectrum of random matrices, Teoret. Mat. Fiz. 10, 102-112 (1973).

[7] T. Tao and V. Vu, Random matrices: Universality of the local eigenvalue statistics, submitted.

Institute of Mathematics, University of Munich, Theresienstr. 39, D-80333 Munich, Germany

E-mail address: lerdos@math.lmu.de

Department of Mathematics, Universidad de Costa Rica San Jose 2060, Costa Rica

E-mail address: alexander.ramirezgonzalez@ucr.ac.cr

Department of Pure Mathematics and Mathematical Statistics, University of Cambridge, Wilberforce Rd, Cambridge CB3 0WB, UK

E-mail address: b.schlein@dpmms.cam.ac.uk

Department of Mathematics, UCLA, Los Angeles CA 90095-1555

E-mail address: tao@math.ucla.edu

Department of Mathematics, Rutgers, Piscataway, NJ 08854

E-mail address: vanvu@math.rutgers.edu

Department of Mathematics, Harvard University Cambridge MA 02138, USA4

E-mail address: htyau@math.harvard.edu 